\title{
Membrane Fluidity and Phospholipid Composition in Relation to Sulfur Amino Acid Intake in Brush Border Membranes of Rat Kidney
}

\author{
RUSSELL W. CHESNEY, NAOMI GUSOWSKI, AND ISRAEL ZELIKOVIC \\ Pediatric Renal Disease Laboratory, Departments of Pediatrics, The University of Wisconsin-Madison, Madison, \\ Wisconsin 53792 and The University of California, Davis, Davis, California 95616
}

\begin{abstract}
The transport of ions and solutes across biological membranes may relate to changes in the lipid microenvironment of the membrane which could alter the activity or configuration of transport sites. Changes in the sulfur amino acid content of diets fed to young rats results in an increase in $\mathrm{Na}^{+}$-taurine symport in brush border membranes isolated from animals fed a low sulfur amino acid diet and a reduction in symport after a high taurine diet in comparison to uptake by membranes from normally fed animals ("the renal adaptive response"). We explored the possibility that diet-induced changes in brush border membrane symport relate to altered membrane fluidity and phospholipid composition in response to diet. An Arrhenius plot of initial rate (15 s) taurine uptake in breakpoint at $22^{\circ} \mathrm{C}$, but no change in relation to dietary alteration. Fluorescence polarization data employing the probe 1-6diphenyl-1,3,5-hexatriene best fits a two-phase linear model employing a computer model fitting program. Dietary manipulations did not change the breakpoint upper segment slope or lower segment slope after incubating fresh membranes with DPH over the temperature range $4-56^{\circ}$ C. No change was evident in membrane phospholipid composition in relation to diet. This study indicates that the changes in initial rate $\mathrm{Na}^{+}$-taurine symport in relation to diet are less likely to be due to changes in the configuration of the transporter from an alteration of the lipid microenvironment of the membrane. (Pediatr Res 20: 1305-1309, 1986)
\end{abstract}

\section{Abbreviations}

LTD, low sulfur amino acid diet NTD, normal sulfur amino acid diet HTD, high taurine diet

DPH, 1,6-diphenyl-1,3,5-hexatriene

tPnA, transparanic acid

TMA-DPH, 1,-(4-trimethylammoniumphenyl)-6-phenyl1,3,5-hexatriene

BBMV, brush border membrane vesicles

BSA, bovine serum albumin

$P$, polarization of fluorescence

PC, phosphatidylcholine

SM, sphingomyelin

Lyso PC, lysophosphatidyl-choline

PI, phosphatidylinositol

Received June 2. 1986: accepted July 25, 1986.

Reprint requests, Russell W. Chesney, M.D., University of California, Davis Medical Center, Department of Pediatrics, 4301 X Street, Sacramento, CA95817.

Supported in part from NIH Grants AM 31682-02 and AM 37223-01.
PS, phosphatidylserine

PE, phosphatidylethanolamine

PG, phosphatidylglycerol

ANOVA, analysis of variance

Renal tubular epithelium is capable of adapting to changes in the dietary intake of various nutrients. This renal adaptive response has been demonstrated for the $\beta$-amino acid, taurine, which is conserved following diminished sulfur amino acid intake and hyperexcreted in the urine after dietary taurine excess (1-3). Studies employing isolated luminal brush border membrane vesicles are consistent with these changes in urinary excretion of taurine occurring as the consequence of a membrane-related event $(3,4)$, since the LTD appears to increase the Vmax of initial $\mathrm{Na}^{+}$-taurine symport and the HTD decrease the Vmax of uptake into vesicles. We conducted these studies to determine if these diets influence the microviscosity and phospholipid composition of these vesicles.

Changes in the physical status or in the lipid composition of biological membranes can alter the movement of ions and solutes across these membranes (5). The technique of fluorescence polarization has been employed to define the "fluidity" of hepatic, erythrocyte, bacterial cell, gastrointestinal, and renal plasma membranes $(6-11)$. The importance of the lipid microenvironment of biological membranes is also of interest since the activity of membrane-bound enzymes and transport-sites can be influenced by alterations in phospholipid composition (11-13). Lipidprotein interactions may impinge on the activity of proteins located within the membrane (13-16). Hence it is tempting to speculate that the renal adaptive response to alterations in sulfur amino acid intake may be due to changes in membrane fluidity and phospholipid composition in relation to dietary change.

In this study we examine the effect of three diets consisting of varying amounts of sulfur amino acids on membrane fluidity, measured by the use of the fluorescence probe DPH over a wide range of temperatures. In addition the influence of these diets on the phospholipid composition of the apical membrane was assessed. These determinations allow us to indicate if changes in brush border membrane accumulation of taurine are governed by alterations in membrane fluidity or phospholipid compositions.

\section{METHODS}

Animals. Male Sprague-Dawley rats (King Animal Labs, Inc., Oregon, WI) aged 58-60 days and weighing 150-180 g were 
employed in all studies. Animals were fasted overnight prior to sacrifice and allowed access to water only.

Diets. The composition of the diet is described in detail elsewhere $(3,4,17)$. Briefly each diet (LTD, HTD, or normal taurine diet (NTD)] is isoproteinic and contains soy protein which contains limited amounts of methionine and cysteine. The LTD contains $0.2 \%$ methionine, the NTD $0.7 \%$ methionine, and the HTD $0.7 \%$ methionine and $3.0 \%$ taurine $(\mathrm{w} / \mathrm{w})$. The methionine content for optimal growth is $0.67 \%$ of diet as methionine (18). Diets are purchased from ICN, Cleveland, $\mathrm{OH}$. Animals are divided into the three dietary groups and consume the diet for 14 days. Evidence for the adaptive response occurs within $72 \mathrm{~h}$ after initiation of diet (17) and is completely established within 6 days. Animals consuming the LTD, which is limiting in methionine and cysteine are $13 \pm 2 \%$ smaller at the end of the 14 days $(3,4,17)$. No attempts are made to pair-feed these animals since the LTD-fed group actually ingests more food.

Preparation of membranes. BBMV are prepared according to a modification of the method of Booth and Kenny (19) as completely described previously (4). The final BBMV fraction was resuspended in $290 \mathrm{mM}$ Mannitol, $10 \mathrm{mM}$ Tris/HEPES (pH 7.4), and either used fresh or frozen at $-70^{\circ} \mathrm{C}$. Protein was measured according to Lowry et al. (20) using BSA as a standard. Determinations were made of membrane $\gamma$-glutanyl transferase, 5 '-nucleotidase, acid phosphatase ( $\mathrm{pH} 4.8$ ), N-acetyl-B-D-glycosaminidase, succinyl-cytochrome $\mathrm{C}$ reductase, malate dehydrogenase, DNA, oubain-inhibitable $\mathrm{Na}^{+}+\mathrm{K}^{+}$ATPase $(3,4)$. Of marker enzymes examined the final membrane preparation contained a 6- to 11 -fold increase in specific activity for $\gamma$-glutamyltranspeptidase and $5^{\prime}$-nucleotidase relative to the starting homogenate and a 1.2- to 1.4-fold increase in $\mathrm{Na}^{+}+\mathrm{K}^{+}$ATPase, a marker of basal-lateral membranes. All other marker enzymes had a lower specific activity indicating excellent enrichment of the brush border fraction.

Uptake studies. The uptake of taurine into brush border membrane vesicles at $23^{\circ} \mathrm{C}$ was performed and according to our established procedure $(3,4)$. Uptake is $\mathrm{Na}^{+}$and $\mathrm{Cl}$-dependent (21) and takes place into an osmotically influenced space, thus accumulation of taurine represents intravesicular uptake (2-4, 21). The internal medium consisted of $278 \mathrm{mM}$ mannitol, $1 \mathrm{mM}$ $\mathrm{Mg} \mathrm{SO}_{4}$, and $10 \mathrm{mM}$ Tris/HEPES (pH 7.4) and the uptake medium (external) consisted of $100 \mathrm{mM} \mathrm{NaCl}, 1 \mathrm{mM} \mathrm{MgSO}_{4}$, $10 \mathrm{mM}$ Tris/HEPES (pH 7.4), and $10 \mu \mathrm{M}$ taurine with $0.5 \mu \mathrm{Ci}$ of ${ }^{3} \mathrm{H}$-taurine. After a given time interval incubated membranes were diluted with a cold $\left(4^{\circ} \mathrm{C}\right)$ stop-solution and filtered over a Millipore filter using a cellulose nitrate membrane (HWAP 0.45 $\mu$ ) after washing three times, membranes were dried overnight, placed in a scintillation vial, submerged in Aquasol, and counted in a $\beta$-scintillation counter with background subtraction $(3,4$, 21).

Fluorescence polarization. For fluorescence polarization determinations, the lipid soluble fluorescent probe DPH (Aldrich Chemical Co., Inc., Milwaukee, WI) was employed in a $2 \mathrm{mM}$ stock solution in tetrahydrofuran. In a usual experiment, vesicles (2-4 $\mathrm{mg}$ protein/ml) in $288 \mathrm{mM}$ mannitol, $10 \mathrm{mM}$ Tris/HEPES $\mathrm{pH} 7.4$, and $1 \mathrm{mM} \mathrm{MgSO}_{4}$ were incubated with $\mathrm{DPH}$ for $15 \mathrm{~min}$ at a final concentration of $4 \mu \mathrm{M}$. P was measured using an Elscint model MV-la microviscosimeter (Elscint Inc., Hackensack, NJ). This device displays $P=\left(I_{11}-I_{1}\right) \div\left(I_{11}+I_{1}\right)$ where $I_{11}$ and $I_{1}$ represent the fluorescent intensities oriented parallel (11) and perpendicular (1) to the path of polarization of the excitation light (22). Interpretation of the data derived utilizes the Perrin equation, which is written: $\mathrm{r}^{\circ} / \mathrm{r}=1+3 \tau / \mathrm{P}$, where $\mathrm{r}^{\circ}$ is the maximal limiting anisotropy (0.362) for DPH (23), $\mathrm{r}$ is the anisotropy, $\tau$ is the excited state fluorophobe lifetime, and $P$ is the rotational relaxation time. $\mathrm{P}$ is usually expressed as the anisotropy parameter $\left[\left(\mathrm{r}^{\circ} / \mathrm{r}\right)-1\right]^{-1}$, where $\mathrm{r}=\left(\mathrm{I}_{11}-\mathrm{I}_{11}\right) \div\left(\mathrm{I}_{11}+\right.$ $21_{1}$ ). Fluorescence polarization was determined from 4 to $56^{\circ} \mathrm{C}$ with readings determined every $2^{\circ} \mathrm{C}$ using freshly prepared BBMV. The temperature of the vesicle suspension was directly determined with a digital thermometer (Digitec model 5810) which is accurate to $0.1^{\circ} \mathrm{C}$.

There is concern over appropriately drawing the shape of the resulting function when in $\left[\left(\mathrm{r}^{\circ} / \mathrm{r}\right)-1\right]^{-1}$ is plotted versus reciprocal absolute temperature, four models were assessed for their ability to fit the data derived. These models were linear, twophase linear, three-phase linear, and quadratic using linear or quadratic regressions where appropriate. The best two- and threephase linear models were derived using a computer program based on the theory of Hudson (24) as designed by Gourley et al. (25). In order to test the adequacy of each model, residual plots were determined. $F$ tests were performed to indicate the appropriateness of a two-phase compared to a linear model and if a three-phase was superior to a two-phase model. Breakpoints and anisotropy parameters were compared in vesicles prepared from rats on each diet. Mean values were compared between diets using two way analysis of variants.

Phospholipid composition. Extraction and analysis of vesicle phospholipids were accomplished by established methods (2628). In general, frozen membranes were thawed and a chloroform:methanol $(2: 1 \mathrm{v} / \mathrm{v})$ extract was prepared and washed free of nonlipid contaminants by the method of Radin (27). Phospholipids were isolated by two-dimensional thin-layer chromatography using an internal standard consisting of $\left[{ }^{14} \mathrm{C}\right]$-dipalmitoyl PC to measure recoveries for each analysis. Lipid extracts were plated on silica gel $\mathrm{H}$ plates; first direction development was performed in a solvent system of chloroform:methanol:water $(65: 45: 4)$. Plates were than air dried for $10 \mathrm{~min}$, rotated $90^{\circ}$, and developed in tetrahydrofuran:methyal:methanol:water (10:6:4:1). This system allows isolation of PC, SM, Lyso PC, PI, PS, PE, and PG. The method of Bligh and Dyer (26) was used to extract lipids from the gel and phosphorus content was quantitated by the method of Perleman et al. (28). The influence of diet on phospholipid composition was compared using Student's $t$ test between groups or ANOVA when indicated.

Supplies. All chemicals used were reagent grade. $\left[{ }^{14} \mathrm{C}\right]$-dipalmitoyl PC and [ ${ }^{3} \mathrm{H}$-]-taurine were purchased from New England Nuclear, Boston, MA. DPH was purchased from Aldrich Chemical Co., Inc., Milwaukee, WI.

\section{RESULTS}

The uptake of taurine $\left(\mathrm{Na}^{+}\right.$-dependent component) was greater in BBMV prepared from animals fed the LTD-34.6 \pm 2.6 (SE) $\mathrm{pmol} / \mathrm{mg}$ protein $/ 15 \mathrm{~s}$-than in NTD vesicles-18.3 $\pm 2.1 \mathrm{p}$ moles/mg protein $/ 15 \mathrm{~s}, p<0.001$. Taurine uptake was lower in HTD-fed vesicles at $11.8 \pm 1.1 \mathrm{pmol} / \mathrm{mg}$ protein $/ 15 \mathrm{~s}$, significantly different from the NTD contol, $p<0.001$ as has been described in our previous studies $(3,4)$.

When $\mathrm{Na}^{+}$-taurine uptake for rats fed the NTD was examined at various temperatures between 4 and $40^{\circ} \mathrm{C}$, an Arrhenius plot can be described (Fig. 1). As noted the best computer fit was a two-limb linear model with a break-point at approximately $22^{\circ}$. Break point values after LTD and HTD were 23.1 and $22.8^{\circ} \mathrm{C}$, respectively. Thus no alteration in the Arrhenius plot was found despite dietary differences.

$\mathrm{P}$ data were assessed by describing Arrhenius-type plots of the $\log \mathrm{P}$ as a function of reciprocal temperature. Residual plots were determined which allows evaluation of the adequacy of the four models being tested to define the best fit. Using this system, in order for a model to be appropriate, there should be no distinct pattern to the residuals plotted; i.e. they should be random. The residual plots for a linear model were parabolic in shape. The two-phase model showed no pattern to its residual plot, whereas a three-phase and quadratic model had marked residual plots. The two-phase linear model also had the lowest residual sum of squares in all 12 data sets. Finally, when $F$ tests were performed to further compare models the two-phase linear model was far superior to the linear or three-phase linear model. A typical plot of $\log \mathrm{P}$ as a function of reciprocal temperature is shown in 
Figure 2. Each figure represents the results of a representative experiment from animals on each of the three diets. The results given in Tables 1 and 2 represent the mean \pm SE of data from all experiments. Thus, the apparent increase in polarization in the LTD animal (Fig. $2 A$ ) is not real when all the data available are analyzed.

Table 1 demonstrates transition temperatures or the temperature at which the segmented log $\mathrm{P}$ versus reciprocal temperature function abruptly changes slope. The transition temperatures show no signficant differences between LTD-, NTD-, or HTDfed rat brush border membranes (ANOVA). Table 2 shows the values for $\mathrm{P}$ and the slopes of the upper and lower segments which represents the apparent energy of activation (10,11). Again no influence of dietary manipulation on these values was evident.

The phospholipid composition of membranes is displayed in Table 3. The most prevalent phospholipids are SM, PC, PS, and $P E$ regardless of diet. No alteration in the phospholipid profile of the membranes could be associated with the dietary change carried out in this study other than the percentage of glycerol-

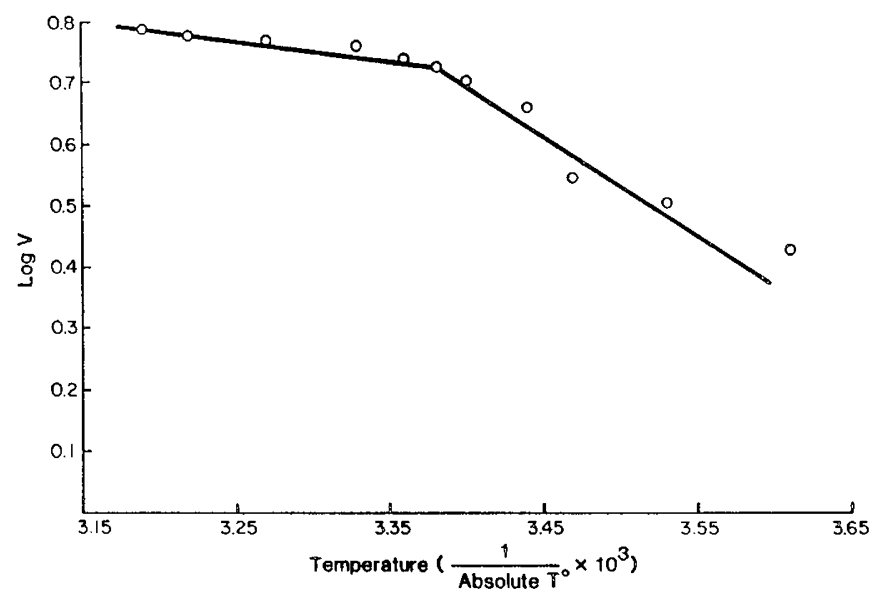

Fig. 1. The initial rate $(15 \mathrm{~s})$ uptake of $10 \mu \mathrm{M}$ taurine in the presence of an external $100 \mathrm{mM} \mathrm{NaCl}$ gradient in relation to temperature (4-56 C). Data are expressed as the reciprocal of the absolute temperature, an Arrhenius plot. Each point represents the mean of five separate experiments determined in triplicate. The plot was fit to a 2-phase linear model using the program established by Gourley et al. (25).
$\mathrm{PO}_{4}$ is slightly but significantly higher in vesicles prepared from rats fed the HTD.

\section{DISCUSSION}

As noted in previous studies (2-4) considerable differences in initial rate $\mathrm{Na}^{+}$-taurine symport are found in this study in relation to dietary intervention. The LTD results in an almost 2-fold increase in 15-s, taurine uptake by BBMV and the HTD in a $6 \%$ fall in uptake. Because of these substantial differences in $\mathrm{Na}^{+}$taurine symport associated with varying intakes of sulfur amino acids it is tempting to speculate that changes in the lipid microenvironment might underlie these changes.

We first determined the breakpoint of a standard Arrhenius plot and showed that a two phase linear model gave the best fit. The breakpoint determined in membranes from rats fed each of the three diets was similar at approximately $23^{\circ} \mathrm{C}$; thus, a dietary effect is not evident. Changes in physical state of the membrane often correspond to liquid-gel phase transitions in a variety of membranes including the intestine $(29,30)$. The observation that a clear change in slope in the Arrhenius plot for $\mathrm{Na}^{+}$-dependent taurine uptake occurs around $23^{\circ} \mathrm{C}$ provides evidence that a liquid $\rightarrow$ gel phase transition may be occurring at this temperature. Further, it is similar to the nonlinear Arrhenius plot for alkaline phosphatase in rabbit kidney brush border membranes which occurs at $26^{\circ} \mathrm{C}(10)$. Whatever are the molecular events responsible for the $\mathrm{Na}^{+}$-taurine symport being responsive to changes in the physical state of the membrane lipids, it is clear that the change in the slope of the Arrhenius plot occurs at "room temperature." Hence, it is timely that all experiments were performed within a narrow range around this temperature. Finally, even though this liquid $\rightarrow$ gel transition may occur at a critical temperature for taurine uptake, the dietary manipulations

Table 1. $P$ data as function of reciprocal temperature $\left(K^{\circ}\right)$ in brush border membranes from rats in each diet

\begin{tabular}{cclc}
\hline Diet & $\mathrm{K}^{*}$ & \multicolumn{2}{c}{ Statistics } \\
\hline LTD & $25.3 \pm 1.1$ & LTD $v$ s NTD & NS \\
NTD & $23.5 \pm 0.6$ & NTD vs HTD & NS \\
HTD & $23.6 \pm 0.1$ & LTD vs HTD & NS \\
\hline
\end{tabular}

* Temperature $\pm \mathrm{SE}$ of five separate experiments expressed as ${ }^{\circ} \mathrm{C}$. Statistics by ANOVA.
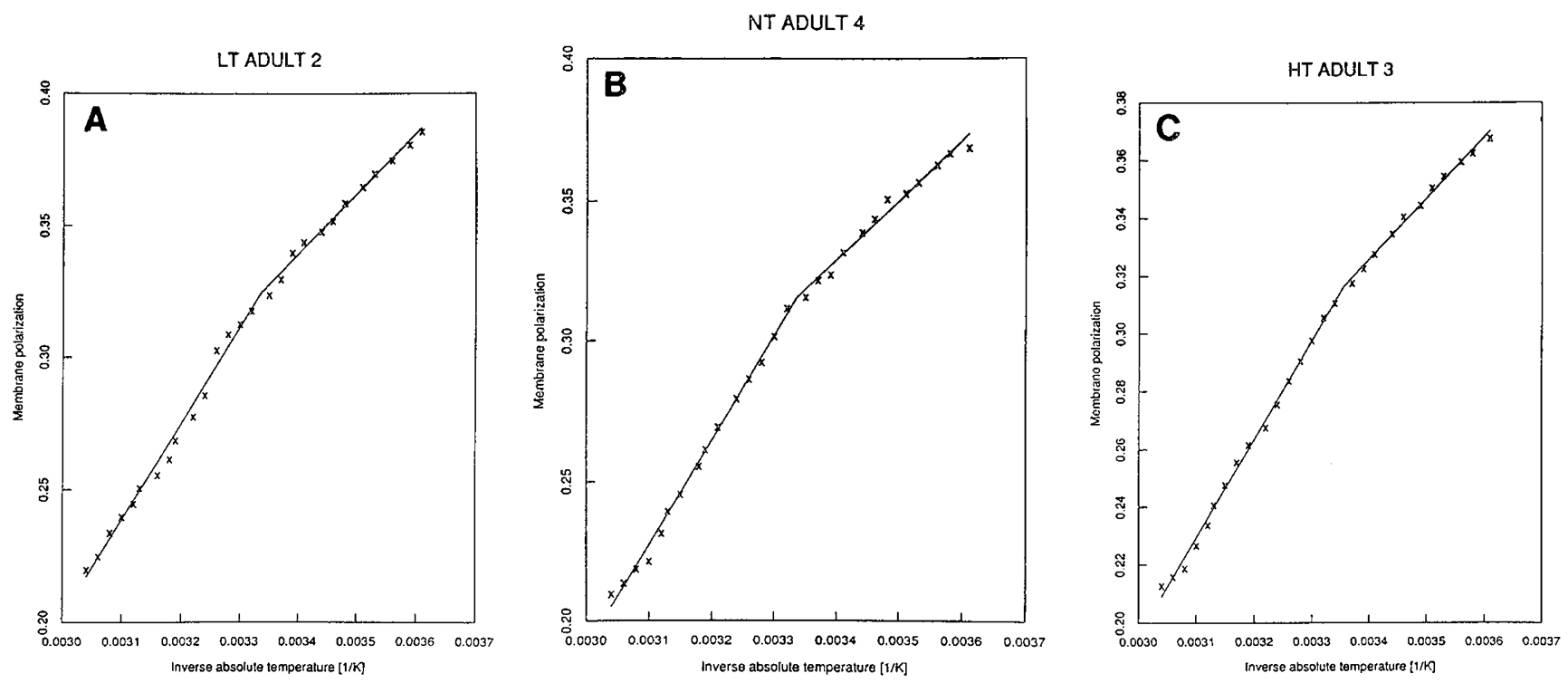

Fig. 2. Computer derived plots of membrane polarization data derived in relation to inverse absolute temperature. $A$, low taurine diet; $B$, normal diet: $C$. HID. Each point is a representative graph of one of four determinations in membranes prepared from animals on each diet. 
Table 2. Values for $P$ and slopes of upper and lower segments

\begin{tabular}{|c|c|c|c|c|c|c|}
\hline & LTD & NTD & HTD & $\begin{array}{c}\text { LTD } \\
\text { vs NTD }\end{array}$ & $\begin{array}{c}\text { NTD } \\
\text { vs HTD }\end{array}$ & $\begin{array}{c}\text { LTD } \\
\text { vs HTD }\end{array}$ \\
\hline$P$ & $\begin{array}{l}0.3366^{*} \\
\pm 0.0106\end{array}$ & $\begin{array}{r}0.3341 \\
\pm 0.0080\end{array}$ & $\begin{array}{r}0.3342 \\
\pm 0.0060\end{array}$ & NS & NS & NS \\
\hline Upper segment & $\begin{array}{r}353.0 \\
\pm 9.1\end{array}$ & $\begin{array}{r}320.33 \\
\pm 23.13\end{array}$ & $\begin{array}{r}341.17 \\
\pm 2.85\end{array}$ & NS & NS & NS \\
\hline Lower segment & $\begin{array}{r}204.88 \\
\pm 32.10\end{array}$ & $\begin{array}{r}170.88 \\
\pm 16.51 \\
\end{array}$ & $\begin{array}{r}196.84 \\
+12.49 \\
\end{array}$ & NS & NS & NS \\
\hline
\end{tabular}

* Mean \pm SE of five determinations at each point. Statistics by ANOVA.

Table 3. Phospholipid composition of brush border membranes*

\begin{tabular}{|c|c|c|c|c|c|c|}
\hline & LTD & NTD & HTD & $\begin{array}{c}\text { LTD } \\
\text { vs NTD }\end{array}$ & $\begin{array}{c}\text { NTD } \\
\text { vs HTD }\end{array}$ & $\begin{array}{c}\text { HTD } \\
\text { vs LTD }\end{array}$ \\
\hline \multirow[t]{2}{*}{ Lyso PC } & 6.86 & 6.78 & 8.20 & NS & NS & NS \\
\hline & \pm 2.18 & \pm 2.18 & \pm 1.88 & & & \\
\hline \multirow{2}{*}{ SM } & 82.60 & 82.27 & 88.23 & NS & NS & NS \\
\hline & \pm 4.30 & \pm 2.30 & \pm 5.78 & & & \\
\hline \multirow[t]{2}{*}{$\mathrm{PC}$} & 71.97 & 62.48 & 78.84 & NS & NS & NS \\
\hline & \pm 8.46 & \pm 12.05 & \pm 2.47 & & & \\
\hline \multirow[t]{2}{*}{ PS } & 113.41 & 133.49 & 113.67 & NS & NS & NS \\
\hline & \pm 6.70 & \pm 11.01 & \pm 7.18 & & & \\
\hline \multirow[t]{2}{*}{ PI } & 3.65 & 2.50 & 2.01 & NS & NS & NS \\
\hline & \pm 0.47 & \pm 1.01 & \pm 0.55 & & & \\
\hline \multirow[t]{2}{*}{ PE } & 63.46 & 60.39 & 67.79 & NS & NS & NS \\
\hline & \pm 5.72 & \pm 0.78 & \pm 6.12 & & & \\
\hline \multirow[t]{2}{*}{ Glycerol phosphate } & 5.12 & 1.27 & 1.55 & $<0.05$ & NS & $<0.05$ \\
\hline & \pm 0.55 & \pm 0.59 & \pm 0.65 & & & \\
\hline
\end{tabular}

${ }^{*} \mu \mathrm{mol} \mathrm{Pi} / \mathrm{mg} ;$ mean $\pm \mathrm{SE}$ of four determinations. Statistics by ANOVA.

that we have employed do not alter this change occurring at "room temperature."

In terms of DPH fluorescence with regard to temperature, the two-phase linear model gave the most appropriate fit. Again regardless of diet, the transition temperature at which the segmented $\log \mathrm{P}$ in relation to reciprocal temperature abruptly changed slope as similar. Since the values for P and the slopes of the upper and lower segment were also uninfluenced by diet, changes in the physical composition, and membrane fluidity (11-15), at least as measured by the fluorescent probe DPH, do not appear to play a role in the renal adaptive response expressed at the apical membrane of the proximal tubule.

Nonuniform distribution of membrane components raises some degree of uncertainty regarding the lipid microenvironment "viewed" by only one fluorescent probe. Investigators have employed in addition to DPH, such probes as tPnA (10) or TMADPH (31). Herein we measured fluidity with only a single probe, DPH, since several of these other probes did not appear to provide a great deal more information. The fluorescence anistropy and transition temperatures found in rabbit renal brush border membranes were almost identical when DPH and tPnA were used in parallel (10). The probe TMA-DPH may be less sensitive in rat renal brush border membranes (31) and, in general, is a less sensitive probe (32). Furthermore, the probe DPH is less influenced by changes in ionic composition (23). Finally since we found no change in transition temperature, $\mathrm{P}$, and in apparent energy of activation in relation to dietary manipulation using DPH as a probe, we purposely chose not to examine other probes. Nonetheless, since we have employed only one probe in $\mathrm{P}$ experiments, we cannot be certain that the changes in diet might not cause a change in the lipid environment of the brush border membrane probed by other compounds.

As a further measure of the lipid microenvironment, we measured the phospholipid composition of the membranes. As indicated in Table 3 , the only change noted in relation to diet was a higher glycerol phosphate value in membranes from LTD-fed animals. Nonetheless this difference was relatively minor in relationship to other phospholipids which were far more prevalent in the membrane such as PC, PS, and PE. Other groups (10, 11) have also reported high levels of these phospholipids in dog and rat BBMV although minor differences in the relative concentrations of each phospholipid species were found between the observations herein and those reported by Molitoris and Simon (11).

It may not be readily apparent why manipulation of amino acids in the diet could, at least theoretically, result in a change in the phospholipid composition of the membrane. One of the main factors that can affect the status of lipids within the plasma membranes is the potential for oxidant stresses within the environment. An important protective system which prevents peroxidation of membrane-bound lipids is reduced glutathione (39). A reduction in the extent of unsaturation of the acyl chains of phospholipids will increase membrane fluidity, lead to phase separations in the lipid domain, and enhance enzyme activity $(10,39)$. The LTD contains limiting quantities of methionine and cysteine which could potentially limit the availability of cysteine for glutathione synthesis and high levels of taurine are known to protect against oxidant stresses (34). Even though we have demonstrated that the glutathione-oxidizing compound, diamide, has no influence on taurine uptake by BBMV (35), the potential for alterations in the diet with reference to sulfur amino acids to alter membrane phospholipid composition by the mechanism of reduced protection against oxidant stress is worthy of investigation. After examining the influence of the three diets on phospholipid composition and on membrane fluidity, this mechanism does not seem likely.

In summary, this study fails to show any change in Arrhenius plot, membrane fluidity, or phospholipid composition as underlying the change in $\mathrm{Na}^{+}$-taurine symport brought about by alterations in the sulfur amino acid composition of the diet. Since the change in symport is expressed as a change in Vmax of initial rate uptake (1-4), an alteration in the amount of $\mathrm{Na}^{+}$-taurine 
transporter is more likely to be the mechanism of the adaptive response rather than a change in the configuration of the transporter. Nonetheless, the present study is limited in that it examines only a single probe of membrane fluidity, and has not examined fatty acid composition of these phospholipids. Accordingly, it is difficult to make a definitive statement regarding the lipid environment around the $\mathrm{Na}^{+}$-taurine transporter.

\section{REFERENCES}

1. Friedman A, Albright PW, Chesney RW 1981 Dietary adaptation of taurine transport by rat renal epithelium. Life Sci 29:2415-2419

2. Rozen R, Scriver CR 1982 Renal transport of taurine adapts to perturbed taurine homeostasis. Proc Natl Acad Sci USA 79:2101-2105

3. Chesney RW, Gusowski N, Friedman AL 1983 Renal adaptation to altered dietary sulfur amino acid intake occurs at the luminal brush border membrane. Kidney Int 24:588-594

4. Chesney RW, Gusowski N, Dabbagh N 1985 Renal cortex taurine content regulates renal adaptive response to altered dietary intake of sulfur amino acids. J Clin Invest 76:2213-2221

5. Jain MK 1980 Phase properties of bilayers. In: Jain MK, Wagner RC (eds) Introduction to Biological Membranes. Wiley. New York,pp 53-175

6. Luly P, Shinitzky M 1979 Gross structural changes in isolated liver cell plasma membranes upon binding of insulin. Biochemistry 18:445-449

7. Massa ME, Moreno RD, Bloj B. Farias RN 1975 Hormone action and membrane fluidity: effect of insulin and cortisol on the Hill coefficients of rat erythrocyte-bound acetylcholinesterase and sodium-potassium-ATPase. Biochem Biophys Res Commun 66:115-122

8. Moreno HM, Farias RN 1976 Insulin decreases bacterial membrane fluidity. Is it a general event in its action. Biochim Biophys Res Commun 72:74-80

9. Gourley GR, Korsmo HA, Olsen WA 1983 Intestinal mucosa in diabetic rats: studies of microvillus membrane composition and microviscosity. Metabolism 30:1053-1058

10. LeGrimellec C, Giocondi MC, Carriere B, Carriere S, Cardinal J 1982 Membrane fluidity and enzyme activities in brush border and basolateral membranes of the dog kidney. Am J Physiol 242:F246-F253

11. Molitoris BA, Simon FR 1985 Renal cortical brush-border and basolateral membranes: cholesterol and phospholipid composition and relative turnover. J Membr Biol 83:207-215

12. Lee AG 1977 Lipid phase transitions and phase diagrams. I. Lipid phase transitions. Biochim Biophys Acta 472:237-281

13. Lee AG 1977 Lipid phase transitions and phase diagrams. II. Mixtures involving lipids. Biochim Biophys Acta 472:285-344

14. Ohyashiki T, Taka M, Mohri T 1985 The effects of ionic strength on the protein conformation and the fluidity of porcine intestinal brush border membranes. J Biol Chem 260:6857-6861

15. Brasitus TA, Schacter D, Mamouneas TG 1979 Functional interactions of lipids and proteins in rat intestinal microvillus membranes. Biochemistry
18:4136-4144

16. Bratsitus TA, Dudeja PK 1985 Alterations in the physical state and composition of brush border membrane lipids of rat enterocytes during differentiation. Arch Biochim Biophys 240:483-488

17. Friedman AL, Albright PW, Gusowski N, Padilla M, Chesney RW: 1983 Renal adaptation to alterations in dietary amino acid intake. Am $\mathrm{J}$ Physiol 245:F159-F166

18. Rogers QR, Harper AE Jr 1965 Amino acid diets and maximal growth. J Nutr 87:267-273

19. Booth AGE, Kenny AJ 1974 A rapid method for the preparation of microvilli from rabbit kidney. Biochem J 142:575-581

20. Lowry DH, Rosenbrough NJ, Farr AL, Randall RJ 1952 Protein measurement with the Folin phenol reagent. J Biol Chem 193:265-275

21. Chesney RW, Gusowski N, Dabbagh S, Theissen NM, Padilla M, Diehl A 1985 Factors affecting the transport of $\beta$-amino acids in rat renal brush border membrane vesicles: the role of external chloride. Biochim Biophys Acta $812: 702-712$

22. Bentley KL, Thompson LK, Klebe RJ, Horowitz PM 1985 Fluorescence polarization: a general method for measuring ligand binding and membrane viscosity. Biotechniques 3:356-366

23. Schachter D, Shinitzky M 1977 Fluorescence polarization studies of rat intestinal microvillus membranes. J Clin Invest 59:536-548

24. Hudson DJ 1966 Fitting segmented curves whose joint points have to be estimated. J Am Stat Assoc 61:1097-1129

25. Gourley GR, Mogilevsky W, Odell GB 1983 Hepatic microsomal composition studies in the Gunn rat. Biochim Biophys Acta 750:419-423

26. Bligh EG, Dyer WJ 1954 A rapid method of total lipid extraction and purification. Can J Biochem Physiol 37:911-917

27. Raden NS 1979 Preparation of lipid extracts. Methods Enzymol 14:245-254

28. Perleman RH, Engle MJ, Kemnitz JW, Kotas RV, Farrell PM 1982 Biochemical and physiological development of fetal rhesus lung. J Appl Physiol 53:230-235

29. Brasitus TA, Tall AR, Schachter D 1980 Thermotropic transitions in rat intestinal plasma membranes studied by differential scanning calorimetry and fluorescence polarization. Biochemistry 19:1256-1261

30. Chapman D, Gomez-Fernandez JC Goni FM 1979 Intrinsic protein-lipid interactions. Physical and biochemical evidence. FEBS Lett 98:211-223

31. Molitoris BA, Alfrey AC, Harris RA, Simon FR 1985 Renal apical membrane cholesterol and fluidity in regulation of phosphate transport. Am J Physiol 249:F12-F19

32. Prendergast FG, Hangland RP, Callahan PJ 1981 1-[4-(Trimethylamino) phenyl]-6-phenylhexa-1,3,5-triene: synthesis, fluorescence properties, and use as a fluorescent probe of lipid bilayers. Biochemistry 20:7333-7338

33. Shinitzky M, Barenholz Y 1978 Fluidity parameters of lipid regions determined by fluorescence polarization. Biochim Biophys Acta 515:367-394

34. Chesney RW 1985 Taurine: biological role and clinical implications. In: Barnes LA (ed) Advances in Pediatrics. Yearbook Publishers, Chicago, pp 1-42.

35. Chesney RW, Gusowski N, Albright P 1985 Inhibition of $\beta$-amino acid transport by diamide does not involve the brush border membrane surface. Pediatr Pharmacol 5:63-72 